\title{
Method to Generate Text Summary by Accounting Pronoun Frequency for Keywords Weightage Computation
}

\author{
Dr. Siddhaling Urolagin, \\ Department of Computer Science and Engineering,Birla Institute of Technology \& Science, Pilani, \\ Dubai International Academic City, Dubai. \\ siddhaling@dubai.bits-pilani.ac.in
}

\begin{abstract}
In recent years large volume of data being generated every day from various sources. Text summarization has become more relevance for quick searching, abstract generating, automatic sorting etc., to larger volume of data. Extractive methods are involve in identifying import part of the text to produce summary. While generating the summary by extractive methods, important keywords are identified by eliminating stopwords. As a part of stopwords removal, pronoun which are used as placeholders for proper nouns in text are usually removed. But frequency information related to pronouns is significant to improve the quality of summary being generated. We propose in this research a method to replace pronouns with their corresponding proper nouns and then compute the frequency of keywords. The keywords weightage has been calculated based on frequency which intern used to extract importantsentences to form the summary. Experiments are conducted on text data collection and a gain ratio is computed to measure improvement in summary generated from pronoun replacement method.
\end{abstract}

Keywords: text summarization, pronoun replacement, keyword weightage.

\section{Introduction}

In recent years huge amount of data being gathered, accessed and utilized by many user in applications such as [1]. These development have increased interest in automatic text summarization, which is intend to produce brief summary of the given text. The interest in text summarization has grown after research on automatic translation[2]. The text summarization has found many applications such as assistingto search documents by providing the overview to user, to obtain headline from newspaper, to provide shorter details of email threads and provide brief medical information to patients, to generate abstracts of scientific articles etc[2][3].

The text summarization includes steps such as topic identification, interpretation and summary generation[4]. In [5] frame work for topic identification is described along with its applications. The Wikipedia graph centrality method has been discussed in [6] for topic identification. The text interpretation intended to provide meaning of text. Different techniques such as direction based text interpretation [7] and ontology based interpretation [8] have is proposed in literature. The text summarization is to produce overview or synopsis of single or multiple input text documents[3]. In literature there are two categories of summarization methods: abstraction and extraction [9]. Abstraction methods endure to construct semantic representation to generate a brief synopsis of text [5][10]. On the other hand extractive methods[11][12] select words, sentences and phrases to form the summary. 
In a given text, words related to a subject around which the text is written will occur more frequently. One of the effective methods for text summarization is based on finding the frequency of the important words and then extract sentences to form the summary [13]. Initially most commonly occurring stopwords are removed from the text and then remaining word are recognized as important words[13]. Pronouns are most commonly used in natural expressing text to indicate proper nouns. During the process of stopwords removal from text, pronouns are usually removed and thereby loosing the frequency information of proper nouns. We propose a summarizing technique in which we replace pronouns with their proper nouns and then frequency of important words areestimated. We retain frequency information of pronouns and their proper nouns thereby improving the quality of summary generated. In the section 2 we discuss the overall working of proposed summarizing techniques. The detailed description of pronoun replacement and then forming the summary is give in section 3 . In section 4, the proposed summarizing method is illustrated with example. Experimental results are given in section 5 , followed by the conclusion in section 6 .

\section{Pronoun to Proper Noun Matching}

The use of pronouns to refer proper nouns is most common in natural expressing text. Analyzing the occurrences of pronouns and their corresponding proper nouns in the text add greater value to summary being generated. In this research a method is proposed to replace pronouns with proper nouns and then generate summary.

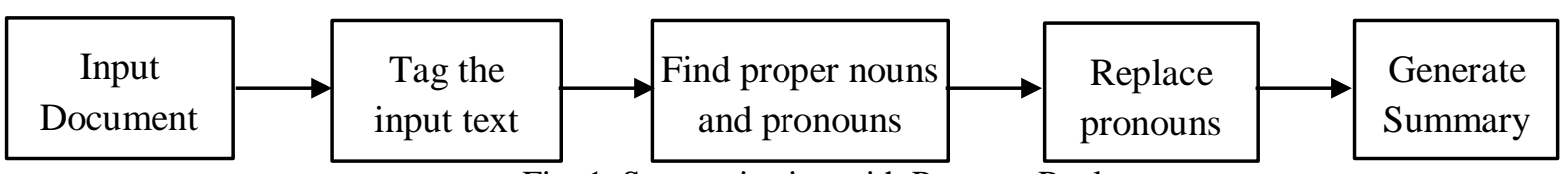

Fig. 1. Summarization with Pronoun Replacement

The approach in extractive method to form summary is to One of the usual approach to form summary is to identify keywords or important words and their frequency in text then form summary by extractive methods[13]. For a given text, at first all stopwords such as the, is, a, that, etc., are removed and remaining words are considered [14][15] as important or keywords. During the process of stopwords elimination, pronouns, which are placeholders for proper nouns also removed and losing significant information. In this research, we propose an approach to generate summary as shown in Fig. 1.Initially the input text is parsed using Part of the Speech (POS) tagger [16][17]. ThePOS takes input sentence and different grammatical parts ofa sentence are identified. Thus recognized pronouns are replaced by proper nouns of an input sentence. While replacing pronoun we substitute most closest preceding proper noun to occurrences of pronouns. Hence, pronouns after replacement are also considered as part of keywords and their frequency information is used while generating the summary. The final summary is formed based on the original input text before replacement of pronouns.

\section{Text Summarization with Pronoun Frequency}

In a given text describing about a topic, words related to that topic will occur more frequently in the text. Thus forming summary based on frequency of words related to topic has found suitable application in several area[18][19] of text analysis. A typical approach to generate summary is to identify important or keywords, which is usually carried out by eliminating stop words such as is, are, a, the, then, etc. A sentence with $n_{s}=$ $n_{k}+n_{o}$ words includes $n_{k}$ number of keywords and $n_{o}$ number of stopwords. The number of times a keyword occurs in the text is determined as frequency $f_{k}$ of the $k^{\text {th }}$ keyword. We compute weightage of $k^{\text {th }}$ keyword with respect to given text as (1). Here $n_{t}$ total number of keywords left after removal of stopwords in the text. 


$$
w_{k}=\frac{f_{k}}{n_{t}}(1)
$$

Before frequency and weight calculation of keywords, we propose to replace pronoun with proper nouns as shown in Fig.2. In natural language describing about a topic, pronouns are more often used to referproper nouns related to topic.

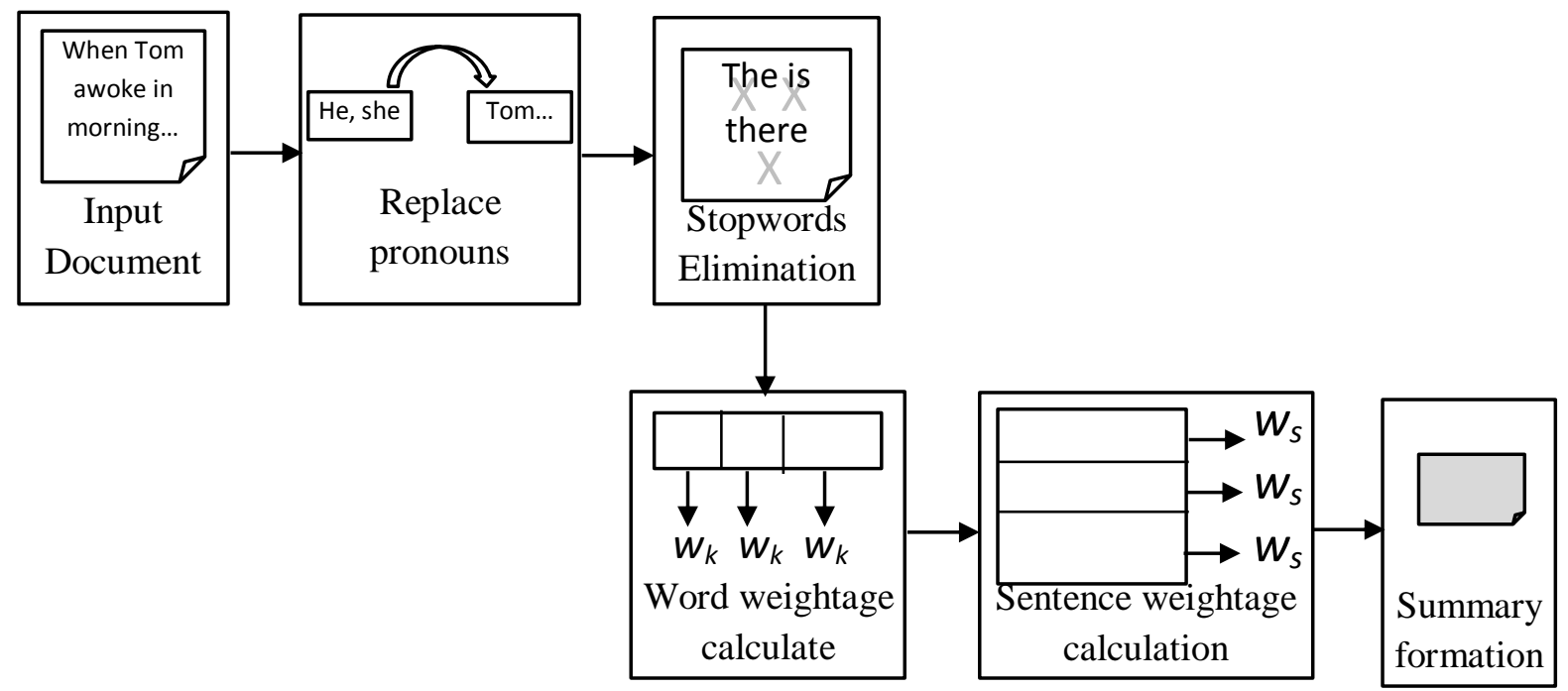

Fig. 2. Automatic text summarization with pronoun frequency.

Once replacement is made in text, we eliminate stopwords then find frequency and weightage of keyword using (1). For a given sentence with $n_{s}$ number of words in it, the sentence weightage $w_{s}$ is computed using(2). Here the sentence contain $n_{k}$ keywords with $w_{k}$ weightage of $k^{\text {th }}$ keyword.A sentence having keywords with more weightage will have higher sentence weightage indicating importance of that sentence in the text.

$w_{s}=\frac{1}{n_{s}} \sum_{k=1}^{n_{k}} w_{k}(2)$

Next, each sentence in the text is assigned an order number starting from one depending on its weightage; the highest weightage sentence assigned one, second highest weightage sentence is assigned two and so on. The summarization ratio is specified by the user, which decides the length of the summary. The length of summary in terms of number of lines $m_{s}$ is calculated based on summarization ratio $s_{r}$ and total number of lines $m_{t}$ in textusing (3).

$m_{s}=\frac{s_{r}}{100} \times m_{t}(3)$

\section{Illustration of Summarization}

The working of proposed summarization method is illustrated on an input text of Fig. 3. In this paragraph 'Siddhartha' is the main topic around which paragraph is written. The pronouns such as 'he', 'his' are used to refer 'Siddhartha' in text several times. An equivalent text generated by replacing all pronouns to their respective proper nouns and then stopwords such as 'when', 'in', 'the' etc., are removed from text. 


\section{Buddha was born in Lumbini about 2500 years ago. His father was king Suddhodhan. His actual name is Siddhartha. He is the founder of Buddhism. He is also called the Light of Asia. Siddhartha was the prince and could enjoy every kind of pleasure. But he did not enjoy the royal pleasure. He was married to Yashodhara and got a son, Rahul. Siddhartha left the palace and his family in search of the truth and reality. He reached to Gaya. He sat under the Peepal Tree and meditated there sitting cross- legged. At last, he gained supreme knowledge. Then he started teaching people about the truth. People started calling him Buddha. He preached his first sermon at Sarnath. His preaching became the norms of religion called Buddhism.}

Fig 3. Example input text

Remaining words are considered as keywords. The Table 1 shows few keywords along with their frequency and weightage found in text of Fig. 3. In the paragraph, 'Buddha' and 'Siddhartha' are proper nouns referred several time using pronouns. With our pronoun replacement technique, the occurrences of pronouns also accounted while finding keywordfrequency. For instance 'Buddha' keyword along with its pronouns has found 9 times in the text.

TABLE1. Keywords frequency and weightage

\begin{tabular}{|l|r|r|}
\hline Keyword & Frequency & Weightage \\
\hline ago & 1 & 0.010989 \\
\hline Buddhism & 2 & 0.021978 \\
\hline Buddha & 9 & 0.098901 \\
\hline Siddhartha & 11 & 0.120879 \\
\hline
\end{tabular}

Thereafter weightage and order of sentences are found. The Table 2 shows sentences after removal of stopwords, number of keywords, weightage and orderin a sentence. The sentence in first row has 8 keywords and its weightage is 0.318681 .Even though number of keywords in sentence of row 3 and row 4 are same, but sentence weightage depends on weightage of keywords. It is noted that sentence weightage of row 3 is more than that of row 4 as shown in Table 2.

TABLE 2. Sentence weightage and order.

\begin{tabular}{|l|r|r|r|}
\hline \multicolumn{1}{|c|}{ Sentence after removing stopwords } & Keywords & Weightage & Order \\
\hline $\begin{array}{l}\text { Siddhartha left palace Siddhartha family search truth } \\
\text { reality }\end{array}$ & 8 & 0.318681 & 1 \\
\hline People started calling Siddhartha Buddha & 5 & 0.274725 & 2 \\
\hline Buddha actual name Siddhartha & 4 & 0.241758 & 3 \\
\hline Buddha father king Suddhodhan & 4 & 0.131868 & 15 \\
\hline
\end{tabular}

The summarization ratio is specified by the user, whichdecides the length of the summary to be generated. Based onthis ratio, the sentences with highest weightage are extracted from original text to form the summary.The Table 3 shows different summary generated depending on summarization ratio. When ratio specified by user is $10 \%$ then two sentences with highest weightage are chosen. For $40 \%$ summarization ratio, six sentences are included in the summary as shown in Table 3. 
TABLE3 Summarization ratio and summary.

\begin{tabular}{|c|c|l|}
\hline $\begin{array}{c}\text { Summarization } \\
\text { Ratio }\end{array}$ & $\begin{array}{c}\text { No. of } \\
\text { lines in } \\
\text { Summary }\end{array}$ & \multicolumn{1}{c|}{ Summarized Text } \\
\hline $10 \%$ & 2 & $\begin{array}{l}\text { Siddhartha left the palace and his family in search of the truth and } \\
\text { reality. People started calling him Buddha. }\end{array}$ \\
\hline $20 \%$ & 3 & $\begin{array}{l}\text { Siddhartha left the palace and his family in search of the truth and } \\
\text { reality. His actual name is Siddhartha. People started calling him } \\
\text { Buddha. }\end{array}$ \\
\hline $40 \%$ & $\begin{array}{l}\text { Siddhartha left the palace and his family in search of the truth and } \\
\text { reality. His actual name is Siddhartha. Siddhartha was the prince and } \\
\text { could enjoy every kind of pleasure.He sat under the Peepal Tree and } \\
\text { meditated there sitting cross- legged. People started calling him Buddha. } \\
\text { He preached his first sermon at Sarnath. }\end{array}$ \\
\hline
\end{tabular}

\section{Experimental Results}

We have prepared text data from various sources such as 100 newspaper articles taken from The Times of India, Deccan Herald, The Hindu and Indian Express, 50 scientific articles and 75 emails. We have given at mostattention to collect wide variety of data. From newspapers different sections are collected ranging from small columns to major stories. For small column articles 2 to 3 paragraphs which have up to 150 to 300 words of text have been collected. The major stories which have made headlines varied from 8 to 10 paragraphs contain approximately up to 600 lines. While collecting scientific text data different section paragraphsare collected for varying length so that text database has wide variety of text to test summarization method.

In order to have comparison, two set of experiments are conducted on the same text documents. At the first, summarization is carried out without the replacement of pronouns. As pronouns are not replaced with their corresponding proper nouns, they also eliminated during stopwords removal process. Then text summary is formed by choosing highest weighted sentences from the text. In the second set of experiments, pronouns are replaced with their corresponding proper noun and the summary is formed. To measure the improvement in quality of summary, we propose a gain ratio matric, which depends on number of keywords present in the summarized text.We define gain ratio as in (5), here $G$ is gain ratio, $n_{c R}$ number of keywords in summary formed after pronoun replacement, $n_{c N}$ number of keywords in summary formed without pronoun replacement.

$$
G=\frac{n_{c R}-n_{c N}}{n_{c N}} \times 100(5)
$$

The Table 4 depicts the results of two set of experiments conducted. In the Table 4, first column shows the input document length, second column and third columns show number of keywords present in the summary. Here the length of input document is determined by number of lines in the text.In forth column gain ratio of (5) is tabulated, showing improvement in summary from first set of experiments to second set with pronoun replacement method.

TABLE4 Gain ratio obtained from two set of experiments.

\begin{tabular}{|c|c|c|c|}
\hline $\begin{array}{c}\text { Input } \\
\text { Document } \\
\text { length }\end{array}$ & $\begin{array}{c}\text { Keywords count } \\
\text { by no pronouns } \\
\text { replacement }\left(n_{c N}\right)\end{array}$ & $\begin{array}{c}\text { Keywords count } \\
\text { by pronouns } \\
\text { replacement }\left(n_{c R}\right)\end{array}$ & Gain\% \\
\hline 7 & 58 & 64 & 10.34 \\
\hline 100 & 445 & 456 & 2.47 \\
\hline 50 & 121 & 125 & 3.30 \\
\hline 250 & 750 & 885 & 18.00 \\
\hline 200 & 610 & 626 & 2.62 \\
\hline
\end{tabular}




\section{Conclusion}

The text describing about a topic, words related to main topic are most frequently used in the text. Therefore, it is reasonable to form text summary based on words frequency. Pronouns are commonly used in the text to refer proper nouns and they are eliminated during stopwords removal process. However, we propose to replace pronouns with their corresponding proper nouns then find weightage of keywords. As pronouns are replaced with proper nouns in text and thereby increasing the frequency of important words.Initially, keywords are identified after eliminating stopwords and then weightage of keywords found based on frequency. Then sentence weightage is computed using keyword weightage. The summary is generated by taking summarization ratio and sentence weightage. Henceforth, the summary generated will include keywords with higher weightage and improving the quality of summary. A gain ratio is define, which indicates percentage improvement of quality of summary generated by pronoun replacement method. The new method of including the pronouns and then form summary has found effective in the experiments conducted to form summary of newspaper article, scientific articles, and email threads.

\section{References}

[1] Fang Chen, Kesong Han and Guilin Chen, "An Approach to sentence selection based text summarization", in Proceedings of IEEE TENCONO2, pp. 489-493, 2002.

[2] Amari, S.-I. and Nagaoka, H. Methods of Information Geometry, "Translations of Mathematical Monographs",in Oxford University Press, 2001.

[3] J. Allan, C. Wade, and A. Bolivar, "Retrieval and novelty detection at the sentence level," in Proceedings of the Annual International ACM SIGIR Conference on Research and Development in Information Retrieval, pp. 314-321, 2003. https://doi.org/10.1145/860435.860493

[4] Hovy, E. and C.-Y. Lin,“Automatic Text Summarization in SUMMARIST” in I. Mani and M.Maybury (eds), Advances in Automatic Text Summarization, pp.81-94. MIT Press, 1999.

[5] Benno Stein, Sven Meyer zu Eissen, "Topic Identification: Framework and Application", in Proceedings of International Conference on Knowledge Management, pp 522-531, 2004.

[6] Kino Coursey, Rada Mihalcea, "Topic Identification Using Wikipedia Graph Centrality”, in Proceedings of NAACL HLT 2009, pages 117-120, Boulder, Colorado, June 2009. https://doi.org/10.3115/1620853.1620887

[7] Marti A. Hearst, "Direction-Based Text Interpretation as an Information Access Refinement", in Text-Based Intelligent Systems, Lawrence Erlbaum Associates, 1992.

[8] Irma Sofia Espinosa Peraldi, Atila Kaya, Sylvia Melzer, Ralf M"oller, "On Ontology Based Abduction For Text Interpretation",in Proceedings of 9th International Conference Computational Linguistics and Intelligent Text Processing, Israel, pp 194-205, 2008.

[9] Lloret, E. \& Palomar, M, “Text summarisation in progress: a literature review", in Artificial Intelligence Review, vol. 37, issue 1, pp 1-41, January 2012. https://doi.org/10.1007/s10462-011-9216-z

[10] Kazuo Sumita, Seiji Miike, Kenji Ono, Tetsuro Chino, "Automatic abstract generation based on document structure analysis and its evaluation as a document retrieval presentation function", in Systems and Computers in Japan, vol. 26, issue 13, Version of Record online: 21 MAR 2007.

[11]Zhang Pei-ying and LI Cun-he "Automatic text summarization based on sentences clustering and extraction", in 2nd IEEE International Conference on Computer Science and Information Technology, pp. 167-168, 2009. https://doi.org/10.1109/iccsit.2009.5234971

[12]Daniel Gayo-avello , Darío Álvarez-gutiérrez , José Gayo-avello, "Naive Algorithms for Key-phrase Extraction and Text Summarization from a Single Document inspired by the Protein Biosynthesis Process", in First International Workshop Biologically Inspired Approaches to Advanced Information Technology, BioADIT 2004, Lausanne, Switzerland, pp. 440-455, January 29-30, 2004.

[13]Rafael Ferreira, Luciano de Souza Cabral, Rafael Dueire Lins, Gabriel Pereira e Silva, Fred Freitas,George D.C. Cavalcanti, Rinaldo Lima, Steven J. Simske, Luciano Favaro, "Assessing sentence scoring techniques for extractive text summarization”, in Expert Systems with Applications,vol.40, issue 14, pp. 5755-5764, 2013. 
https://doi.org/10.1016/j.eswa.2013.04.023

[14] Hassan Saif, Miriam Fernandez, Yulan He, Harith Alani, "On Stopwords, Filtering and Data Sparsity for Sentiment Analysis of Twitter", in LREC, pp. 810-817, 2014.

[15] Stuart Rose, Dave Engel, Nick Cramer, and Wendy Cowley, "Automatic keyword extraction from individual documents", in Text Mining, pp. 1-20, 2010. https://doi.org/10.1002/9780470689646.ch1

[16] Kristina Toutanova and Christopher D. Manning,"Enriching the Knowledge Sources Used in a Maximum Entropy Partof-Speech Tagger", in Proceedings of the Joint SIGDAT Conference on Empirical Methods in Natural Language Processing and Very Large Corpora pp. 63-70. 2000. https://doi.org/10.3115/1117794.1117802

[17] Kristina Toutanova, Dan Klein, Christopher Manning, and Yoram Singer,"Feature-Rich Part-of-Speech Tagging with a Cyclic Dependency Network", in Proceedings of HLT-NAACL,pp. 252-259, 2003. https://doi.org/10.3115/1073445.1073478

[18] José M. Perea-Ortega, Elena Lloret, L. Alfonso Ureña-López, Manuel Palomar, “Application of Text Summarization techniques to the Geographical Information Retrieval task", in Expert Systems with Applications, vol. 40, issue 8, pp. 2966-2974.2013.

[19] Nongnuch Ketui, Thanaruk Theeramunkong, Chutamanee Onsuwan, "An EDU-Based Approach for Thai MultiDocument Summarization and Its Application", in Journal ACM Transactions on Asian and Low-Resource Language Information Processing TALLIP, vol. 14 issue 1, January 2015. 